\title{
Search for binary central stars of the Magellanic Clouds PNe
}

\author{
Marcin Gładkowski ${ }^{1}$ Marcin Hajduk ${ }^{2}$ and Igor Soszyński ${ }^{3}$ \\ ${ }^{1}$ Nicolaus Copernicus Astronomical Center, Polish Academy of Sciences, \\ ul. Rabiańska 8, 87-100 Toruń, Poland \\ email: seyfert@ncac.torun.pl \\ ${ }^{2}$ National Science Centre, \\ ul. Królewska 57, 30-081 Kraków, Poland \\ ${ }^{3}$ Obserwatorium Astronomiczne, Uniwersytet Warszawski, \\ Al. Ujazdowskie 4, 00-478, Warszawa, Poland
}

\begin{abstract}
The Optical Gravitational Experiment (OGLE) was effectively used in discovering binary central stars of planetary nebulae (CSPNe). About 50 binary CSPNe have been hitherto identified in the Galaxy, almost half of them were detected in the OGLE database. We used the OGLE data to search for binary CSPNe in the Magellanic Clouds. We also searched for PNe mimics and removed them from the PNe sample. Here, we present results of the photometric analysis for Small Magellanic Cloud (SMC) and our progress on search of binary central stars in the Large Magellanic Cloud (LMC). So far, we have discovered one binary central star of the PN beyond the Milky Way, which is located in the Small Magellanic Cloud.
\end{abstract}

Keywords. techniques: photometric, binaries: general, planetary nebulae: general, Magellanic Clouds

\section{Introduction}

The Optical Gravitational Experiment (OGLE) survey was originally dedicated to search for microlensing events. However, the massive photometric surveys provide an excellent opportunity to study many other objects and phenomena such as planetary nebulae (PNe). About 50 binary CSPNe have been hitherto identified in the Milky Way, and almost half of them were discovered using the OGLE data. The number of binary CSPNe is growing, thus it is possible to gain the knowledge about the influence of the common envelope phase of the binary evolution on the morphology of the PNe (De Marco 2009; Miszalski et al. 2009b; Boffin 2012). The fraction of close binary CSPNe was established as $12-21 \%$ on the basis of the OGLE photometry for the Galactic bulge (Miszalski et al. 2009), and is consistent with the fraction given by Bond (2000).

\section{Methods of analysis}

PNe for the Magellanic Clouds were selected using SIMBAD database, and then the object type and coordinate search criteria. This allowed us to search the objects. Later on, we update our list by adding the objects, which were missing in the SIMBAD database.

The objects were identified by visual comparison of the finding charts, which were available in the literature with the OGLE II-IV I-band reference images. In addition, we observe $27 \mathrm{PNe}$ in the SMC with the $1.0 \mathrm{~m}$ telescope at South African Astronomical Observatory (SAAO) with the $\mathrm{R}$ and $H_{\alpha}$ filters to confirm some not certain identifications.

We analysed the OGLE-II and -III I-band photometry in the case of the SMC (Hajduk, Gładkowski \& Soszyński 2014), and OGLE-III-IV I-band photometry for the LMC using 


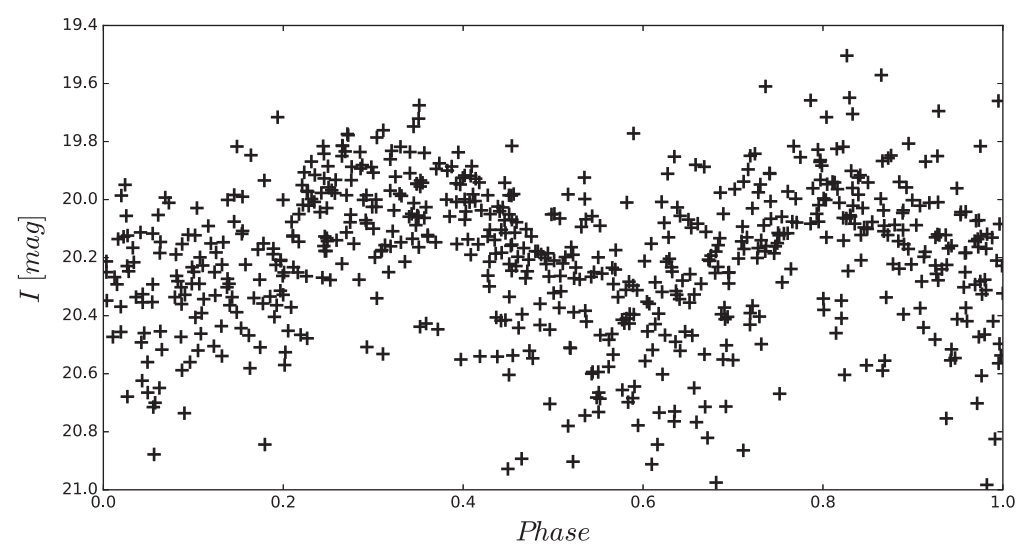

Figure 1. Phased OGLE I-band lightcurve of the Jacoby SMC 1.

the programs PERIOD04 (Lenz et al. 2005) and PERANSO (www.peranso.com). This software offers a variety of period analysis methods from which we chose the analysis of variance (ANOVA, Schwarzenberg-Czerny 1996).

\section{Results}

In a case of the SMC we identified 52 objects in the OGLE reference images in total out of 119 candidates. Eight object were find to be variable: six periodic and two irregular objects. We made the spectroscopic observations of five variables with the Southern African Large Telescope (SALT), and another 3 objects were observed using the Radcliffe $1.9 \mathrm{~m}$ telescope at SAAO. Only Jacoby SMC 1 (see Fig. 1) has a binary CS with a period of 1.16 days assuming the irradiation effect as a cause of the variations, or twice that period in the case of ellipsoidal variations (Hajduk, Gładkowski \& Soszyński 2014). The rest of objects turned out to be the PNe mimics.

For the LMC we have identified about 170 objects in the reference images in total of about 800 candidates so far. The work is in progress. We also found four periodic variables. Unfortunately, none of them is a close binary CSPN.

\section{Conclusions}

So far, we have discovered only one close binary central star of PN beyond our Galaxy - Jacoby SMC 1, which is located in the SMC. We also discovered a new symbiotic star in the SMC: [JD 2002] 11, which was study in details by Hajduk et al. (2015).

\section{References}

Boffin, H. M. J., Miszalski, B., Rauch, T., et al. 2012, Science, 338, 773

Bond, H. E. 2000, ASP Conf. Ser., 199, 115

De Marco, O. 2009, PASP, 121, 316

Hajduk, M., Gładkowski, M., \& Soszyński, I. 2014, A\&̊A, 561, A8

Haberl, F. \& Sasaki, M. 2000, A\& A, 359, 573

Hajduk, M., Gromadzki, M., Mikołajewska, J., Miszalski, B., \& Soszyński, I. 2015, Acta Astronomica, 65, no 2, 139-149

Lenz, P. \& Breger, M. 2005, Comm. Asteroseismol., 146, 53

Miszalski, B., Acker, A., Moffat, A. F. J., Parker, Q. A., \& Udalski, A. 2009, A\& A, 496, 813-825

Miszalski, B., Acker, A., Parker, Q. A., \& Moffat, A. F. J. 2009, A $\& A, ~ 505,249$

Schwarzenberg-Czerny, A. 1996, ApJ, 460, L107 